\title{
Germanica
}

\section{Ingeborg Bachmanns Todesartenzyklus und das Thema Auschwitz}

Peut-on écrire des romans après Auschwitz? Le cycle d'Ingeborg Bachmann

Formes de mort et le thème d'Auschwitz

Irene Heidelberger-Leonard

\section{OpenEdition}

\section{Journals}

Édition électronique

URL : http://journals.openedition.org/germanica/2225

DOI : $10.4000 /$ germanica.2225

ISSN : 2107-0784

\section{Éditeur}

Université de Lille

\section{Édition imprimée}

Date de publication : 1 décembre 1994

Pagination : 189-202

ISSN : 0984-2632

Référence électronique

Irene Heidelberger-Leonard, «Ingeborg Bachmanns Todesartenzyklus und das Thema Auschwitz »,

Germanica [Online], 14 | 1994, Online erschienen am: 01 Januar 1994, abgerufen am 06 Oktober 2020.

URL : http://journals.openedition.org/germanica/2225; DOI : https://doi.org/10.4000/germanica.2225

Ce document a été généré automatiquement le 6 octobre 2020.

(c) Tous droits réservés 


\title{
Ingeborg Bachmanns Todesartenzyklus und das Thema Auschwitz
}

\author{
Peut-on écrire des romans après Auschwitz? Le cycle d'Ingeborg Bachmann \\ Formes de mort et le thème d'Auschwitz
}

Irene Heidelberger-Leonard

1 Streng genommen ist jedes Wort fragwürdig an der Formulierung dieser Fragestellung: Bachmanns Todesartenzyklus gibt es nicht, es gibt ihn nur als Projekt, und das «Thema» Auschwitz ist kein Thema, und sicherlich kein greifbares in Bachmanns Romanen: nirgends findet man bei ihr eine realistische, geschweige denn eine dokumentarische Beschreibung dieser Otschaft. In keinem ihrer literarischen Texte fällt - wenn ich mich nicht irre - selbst der Name dieses Ortes. Bachmann schreibt nicht über Auschwitz, sie schreibt durch Auschwitz hindurch, ja, ich würde sogar so weit gehen: in den Entwürfen Der Fall Franza, Requiem für Fanny Goldmann, in ihrem Roman Malina - schreibt sie Auschwitz, sie schreibt, um es noch deutlicher zu sagen, ihr Auschwitz. Die eigene Geschichte wird ihr zur Geschichte aller, «die doch die grosse Geschichte ausmachen» ${ }^{1}$. Dass eine derart persönliche Auseinandersetzung, ja eine solch private Vereinnahmung dieser weltgeschichtlichen Katastrophe ihrerseits wieder problematisch ist, zumal sie vonseiten der nachgeborenen Tätergeneration vorgenommen wird, lässt sich unschwer erahnen. Denn die Perspektive, die die Autorin dabei in ihren Werken einnimmt, ist primär die der Opfer, und ihre Figuren, deren Vernichtung sie minutiös nachzeichnet, denunzieren die Täterschaft der Mörder.

Was aber kennzeichnet «ihr» Auschwitz? Auschwitz ist für Bachmann in der Tat identisch mit der Stätte, an der Millionen von Juden ausgerottet wurden. Von diesem Auschwitz aber kann sie nur auf vermittelte Art schreiben, weiss sie doch um die Unmöglichkeit seiner realistischen Abbildung. So wird ihr in in ihrer persönlichen Aneignung dieser Name zur Chiffre von Vernichtung schlechthin. Von Verbrechen und Mord, die zwar unter dem Nationalsozialismus ihren anerkannten Höhepunkt hatten, aber in den sogenannten Friedenszeiten, lediglich in einem unterschwelligen NS- 
Kontinuum unerkannt weiter florier en. Herkömmliche Faschismustheorien liest sie gewissermassen gegen den Strich; sie liest sie nicht historisch, sondern geschlechtsspezifisch. Nicht die nationalsozialistische Terror-Herrschaft ist ihr der Ursprung für den Krieg im Frieden, vielmehr sieht sie im Nationalsozialismus eine besonders barbarische öffentliche Variante eines - wie sie ihn nennt - «privaten» Faschismus, der schon seit Anbeginn der Menschheit das Verhältnis zwischen den Geschlechtern unterhöhlt. Als Franzas Bruder Martin den Ehemann Jordan mit dem Faschismus assoziiert, antwortet Franza: «Du sagst Faschismus, das ist komisch, ich habe das noch nie gehört als Wort für ein privates Verhalten [...] Aber das ist gut, denn irgendwo muss es ja anfangen, natürlich, warum redet man davon nur, wenn es um Ansichten und öffentliche Handlungen geht $»^{2}$.

3 Bachmanns Texte geben zu verstehen, dass nicht etwa der «öffentliche» Faschismus sich auf die "privaten» Beziehungen übertragen habe, sondern, im Gegenteil, der «Krieg» zwischen Mann und Frau Urzelle ist für die politischen Monstrositäten des Nationalsozialismus. Eine derartige chronologische Umkehrung hat eine entsprechende gefährliche terminologische Verwischung der Grenzen zur Folge: So legt Bachmanns Metaphorik nämlich eine Gleichsetzung des geschichtlichen Genozids der Juden mit dem jahrhundertsalten «Genozid der Frau» nahe. Nicht weniger problematisch scheint mir Bachmanns symbolische Identifizierung mit den ermordeten Juden. Wieweit, fragt man sich da, ist Einfühlung in nicht selbst erlittene konzentrationäre Erfahrung ästhetisch rekonstruierbar? Wann wird diese Einfühlung zur Lüge? Aber nicht Bachmanns Legitimation soll uns hier primär beschäftigen ${ }^{3}$, sondern das «Was» und das «Wie» ihrer Auseinandersetzung.

4 Dem «Was» kommt man vielleicht am ehesten von aussen bei; man sollte sich Bachmanns Selbstaussagen zu diesem Themen-Komplex noch einmal ins Gedächtnis rufen ${ }^{4}$. Legendär geworden ist in diesem Zusammenhang die Formulierung ihrer «ersten Erinnerung», die sie im Dezember 1971 - im Umfeld also von der Niederschrift von Malina - als ihr Urtrauma vorstellt. Der Einmarsch von Hitlers Truppen in Klagenfurt habe ihre Kindheit zertrümmert, sie verbinde mit dieser Brutalität «das Aufkommen ihrer ersten Todesangst», "einen zu frühen Schmerz», den sie vielleicht «in dieser Stärke später [...] nie mehr hatte» ${ }^{5}$. So fällt der Beginn ihrer Ichfindung und ihres Sozialisationsprozesses mit dem Beginn ihrer IchzerStörung zusammen. Retrospektiv stellt Bachmann auch ihre Promotion als eine Dissertation «gegen» Heideggers «Irrationaldenken» und dessen Komplizenschaft mit Hitlers Diktatur dar ${ }^{6}$.

5 Als sie schliesslich 1973 bei einem ersten Polen-Besuch die Gedenkstätten von Auschwitz und Birkenau aufsucht, verschlägt es ihr buchstäblich die Sprache ${ }^{7}$. Sie habe seitdem jeden Tag «Angstträume und Alpträume».

6 Von ihren halluzinatorischen Transformationen der Geschichte, von der literarischen Umsetzung dieser «Angst-und Alpträume», von den Wortbildern, ja der höchst persönlichen Mythologie, die die bedrohende Intensität der Todesarten-Prosa. ausmachen, soll hier die Rede sein. Angst, empört sich Franza, habe nichts mit dem «Abrakadabra der Philosophen» zu tun, die die Metaphysik bemühen. «Die Angst ist kein Geheimnis, kein Terminus, kein Existential, nichts Höheres, kein Begriff [...]. Die Angst ist nicht disputierbar. Sie ist der Terror, der massive Angriff auf das Leben?

7 Produktionsgeschichtlich gesprochen sollte Der Fall Franza den Zyklus eröffnen, ein Exempel sollte er statuieren, ein dreifaches zumal: Individualpsychologisch, eine Frau wird von ihrem Ehemann zu einem «Fall» entwürdigt; sozialpsychologisch, die 
Vernichtung dieser Frau steht stellvertretend für die Vernichtung «aller» Frauen von «allen» Männern; und ästhetisch, der Fall Franza stellt die Weichen für die zwei folgenden «Fälle», für den «Fall» Fanny Goldmann und für den «Fall» des Malina-Ich. Wie eng Bachmann Franzas Ausmerzung mit der Ausmerzung-spolitik der Nationalsozialisten verknüpft, macht sie in ihrer 1966 gehaltenen Vorrede ${ }^{10} \mathrm{zu}$ diesem Romanfragment deutlich. Das Buch, hören wir, ist ein Buch über ein Verbrechen. Die Massaker seien zwar vorbei, die Mörder aber noch unter uns, auch wenn ihre Mordschauplätze sich nicht mehr stolz zu erkennen gäben, auch wenn die Mörder nicht mehr mit Orden bedacht würden. Die Literatur habe die Aufgabe, «einen ersten Beweis zu erbringen, dass noch heute sehr viele Menschen nicht sterben, sondern ermordet werden $»^{11}$. Heute fänden die Dramen im Innen statt, «kraft der Dimension», ergänzt Bachmann, «die wir oder imaginierte Personen diesem Leidenmachen und Erleiden verschaffen können ${ }^{12}$. Franzas Krankheit und Tod ist nichts weniger als eine Demonstration der Gegenwart der Vergangenheit, wobei die Autorin, wie mir scheint, manchmal in Gefahr gerät, die «inwendigen Verbrechen» der Restauration, wie sie sie nennt, mit dem gezielten Terror der Nazis gleichzusetzen.

Dieser Gefahr war sie m.E. tatsächlich schon in ihrer Erzählung Drei Wege zum See erlegen, wo sie assoziativ die Überlegungen des realen KZ-Häftlings Jean Améry zur physischen Tortur in Verbindung bringt mit den psychischen Nöten ihres Protagonisten Trotta aus Joseph Roths Kapuzinergruft. Dies scheint mir deshalb nicht legitim, weil sie dadurch Amérys Erfahrungen banalisiert, indem sie sie an den geschichtlich viel weniger beschädigten Trotta delegiert. Bedenklich scheint mir ihre Verfahrensweise, Amérys gelebtes Erleiden des Zivilisationsbruchs Auschwitz in ein schon vorgeprägtes Muster zu pressen - Roths Trotta war weder Jude, noch ein politisch Verbannter - und somit paradoxerweise Améry in eine literarische Tradition einzureihen, die doch gerade mit ihm auf eklatante Weise abgebrochen ist ${ }^{13}$.

9 Anders verhält es sich in den Todesarten-Schriften: dort greift Bachmann nirgends auf schon vorgefertigtes Material zurück, sondern löst genau das ein, was sie in ihrer Franza-Vorrede zu leisten verspricht, indem sie sich nämlich durch ihre imaginierten Personen ihr höchst eigenes Leiden er schreibt. Und Franzas Leiden ist die «Angst», eine Angst, die sich geschichtlich verwurzelt weiss. Der Krieg wird ausdrücklich als ihre «Krankheit von Damals» ${ }^{14}$ bezeichnet, als «Einsturz». Ihren Namen von «Damals» will die Kranke von Heute wieder an sich nehmen. An ihm will sie nun festhalten - zur grossen Irritation Martins, der von seiner Vergangenheit nichts mehr wissen will. Das Kriegsende, den Frieden erlebt die Fünfzehnjährige wie ein mystisches Erlebnis: Die Besetzer sind ihr die Befreier, die Freier, die Leben und Liebe versprechen. Der Frieden heisst Sire, ein englischer Captain ${ }^{15}$ und ihm möchte sie sich hingeben. Aber der Captain hat anderes im Sinn und vertröstet die Heranwachsende beim Abschied mit eiligen Küssen, die sie die «englischen Küsse» tauft. «Damit endete Franzas erste Liebe und das ganze Strahlen hörte auf in ihr ${ }^{16}$. Hier also hat ihr Ende angefangen. Der nächste, der ihr «Strahlen» gestohlen hat, ist der Psychiater Jordan, ihr Mann «mit blütenweissen Hemden und Professorentitel». Mit ihm lebt sie in einer Blaubartehe mit «Würgmal am Hals» ${ }^{17}$, in einem «Dschungel, inmitten der Zivilisation» ${ }^{18}$, bevor er sie mit seinen Waffen ${ }^{19}$, mit den «Folterwerkzeugen der Intelligenz» ${ }^{20}$ ermordet. Die zarte Geschichte mit den englischen Küssen, die sie ihm anvertraut hatte, findet sie zu einer klinischen Fallgeschichte aufgearbeitet ${ }^{21}$. «Er ist das Exemplar, das heute regiert, [...] das von heutiger Grausamkeit (ist), das angreift und darum lebt [...], das Raubtier dieser Jahre» $[. . .]^{22}$. Martin bezeichnet ihn wie schon erwähnt wurde als Faschisten, als 
«privaten» Faschisten, der sich ausgerechnet mit einem Buch «Über die Versuche an weiblichen Häftlingen und ihren Spätschäden ${ }^{23}$ einen Namen gemacht hat. Franziska aber, Mitautorin dieses Buches, findet nirgends Erwähnung in diesem Buch. Ihren Namen hat er ausgelöscht. Sie begreift zum ersten Mal, dass Jordan es auf ihre Vernichtung abgesehen hat. Hier, in diesem Moment der Erkenntnis, überfällt sie der nackte Terror der Angst. Auch die Helle der Wüste, die auf Heilung hoffen lässt, verkehrt sich in die dunkelste Finsternis, durchtränkt von Gewalt. Der Wiederholungszwang der Geschichte: ob in dem Bild der gefesselten Frau, ob bei der Hochzeit und dem Kamel mit der durchgeschnittenen Kehle, ob in dem Auskratzen des Gesichts der Königin Hatschepsut, die patriarchalische Brutalitat bleibt unbezwingbar. Jegliches Entrinnen erweist sich als Illusion. Franza ersucht den früheren EuthanasieArzt Dr. Körner, ihr die befreiende Spritze zu geben, die er, Dr. Körner, zwanzig Jahre zuvor zur «Ausmerzung unerwünschten Volkstums»" freiwillig verabreichte.

Franziska Ranner, Nichtzeugin, NichtJüdin, sie, Nachgeborene der Tätergeneration, deren Vater in El Alamein für die Nazis gefallen ist, kann die Vergewaltigungen, die «Wiederholungen ihres Ermordetseins» nur ausdrücken, indem sie sich selbst als Häftling, als Jüdin projiziert, eine Tote auf Abruf, indem sie sich - in ironischer Anlehnung an jordanische Wissenschaftstradition - zum Spätschaden erklärt. Es erscheint schon hier die Vision, die das zweite Kapitel von «Malina» beherrschen wird. «Der Friedhof der Töchter» ${ }^{25}$, der Töchter von «niedriger Rasse». Und wieder verschmelzen sich die Ebenen. Alle Schandtaten des «Raubtier Mensch» verdichten sich der Träumenden in diesem einem Urbild der Barbarei: «Heut nacht hab ich geträumt, ich bin in einer Gaskammer, ganz allein, alle Türen sind verschlossen, kein Fenster, und Jordan befestigt die Schläuche und lässt das Gas einströmen $»^{26}$. Jordan, der Henker von heute, der Körner von gestern: Franza, hilflos, beobachtet ihn bei seinen Vorkehrungen, sie zu töten, mit Gas. Die Gaskammer steht hier als Chiffre für den Raum der Geschichte ${ }^{27}$, für den Raum, wo in Franzas Wahrnehmung die persönliche Geschichte ihrer Vernichtung mit der Vernichtung aller «Minderwertigen» in der «grossen» Geschichte zur Deckung kommt. Der Traum, befreit von den nachprüfbaren Gesetzen der Kausalität, folgt seiner eigenen Schmerzenslogik, lässt die Bilder aufsteigen, die am anschaulichsten, seine Kondition als Opfer versinnbildlichen. Aber sobald der Traum von Bewusstsein gesprengt wird, setzt die Logik der Nacht aus und wird verdrängt von gesellschaftlich antrainierten Rationalisierungen. «Wie kann ich sowas träumen, wie kann ich nur» wirft sich Franza vor, und fügt hinzu: «gleich möchte man um Verzeihung bitten $»^{28}$. Mit dieser letzten Beschwichtigungsformel könnte das pervertierte Verhältnis zwischen Opfer und Täter im Geschichtsraum des Nach-Auschwitz kaum ironischer formuliert werden. So weit ist es tatsächlich gekommen im Nachkriegsdeutschland und - Österreich, dass die Opfer ihre Täter um Vergebung bitten. So wie Franza sich innerlich bei Jordan entschuldigt, ihn in ihrer Imagination einer solchen Mordtat für fähig gehalten zu haben, so wie sie sich später auch beim ex SS-Hauptsturmführer Dr. Kurt Körner ${ }^{29}$ entschuldigt, so entschuldigte sich vor ihr der historisch authentische von ihr zitierte Zeuge B. im Nürnberger Prozess vor seinen Richtern, als er bei seiner Aussage in Schweigen stürzt, bis er schliesslich mit Mühe fortfährt: «Verzeihen Sie, dass ich weine» ${ }^{30}$. Sonst, hält Franza in ihrem Lektürebericht über die Nürnberger Prozesse fest, war in den ganzen Protokollen kein "Verzeihen Sie» vorgekommen, kein einziges Mal von den an den medizinischen Versuchen beteiligten Ärzten, die sich entweder hinter rechtsverbindlichen Formeln 
oder Unwissenheit verschanzten: «niemals brach ein Schweigen aus, nie kam etwas ins Stocken $»^{31}$.

11 Franza durchschaut - erst spät - dass auch sie an diesen Beschwichtigungsmechanismen teilgehabt hat. Sie begreift, Schritt für Schritt, dass auch sie nicht ganz unschuldig ist, dass auch sie be- und verfangen war in den Vergessensstrategien der Nachkriegszeit. Der Moment der Einsicht, in dem sie das volle Ausmass ihres Opferdaseins begreift, macht sie auch sehend für ihre Mittäterschaft. Das willentliche Ausblenden, die bewusste Beschränkung ihrer Wahrnehmungen, erkennt sie, machen sie bei allem Erleiden, auch zur Komplizin ihrer Zerstörung. Erst spät, zu spät wird sie sehend. Und als Sehende kommt sie, wie der Zeuge B., ins Stocken, stürzt sie nicht nur ins Schweigen, sondern erwirkt sie ihr endültiges Verstummen.

Auch Fanny wird zum Verstummen gebracht. Franza und Fanny sind die Gebenden, Jordan und Marek die Nehmenden. «Private Faschisten» sind sie beide; wie Metzger weiden die Männer ihre Frauen aus. Marek wie Jordan ist ein skrupelloser Tyrann, beide sind sie, um mit Bachmann zu sprechen, «Musterschüler der Zeit». Der Fall Franza zeichnete sich aus durch eine klare dualistische Struktur: Frau und Mann, Afrika und Europa, die magischen Schwarzen und die raubgierigen Weissen.

13 Die Figurenkonstellation von Opfer und Täter scheint sich zunächst in Requiem für Fanny Goldmann gleich zu bleiben. Und doch scheinen mir, von evidenten Parallelen abgesehen, gravierende Unterschiede zu bestehen. Wohl wissend, dass ich hier völlig ungesichert Schlüsse ziehe, die, bedenkt man den Fragmentcharakter des Textes ${ }^{32}$, unsicher sind, höre ich aus dem unvollendeten Requiem für Fanny Goldmann einen distanzierteren Ton sprechen im Vergleich zur identifikatorischen Sichtweise im Franza-Fragment. Im Requiem wird narrativisch nicht mehr zwischen «sie», «ich» und «wir» experimentiert, sondern durchgehend in der dritten Person berichtet und zwar von einem Erzähler, der es besser weiss. So sind Franzas und Fannys «Krankheit zum Tode» tatsächlich ganz anders angelegt, sowohl in ihrem Selbstverständnis, als auch, und vor allem -, in ihrer geschichtlichen Kontextualisierung. So sehr Franza nicht nur Jordans Gewalttätigkeit, sondern auch ihre eigene Opferwilligkeit kritisch durchleuchtet, so fraglich ist es, ob Fanny nicht bis zum Schluss ihrer allzu beschränkten Wahrnehmungsfähigkeit verhaftet bleibt. Gewiss werden sie beide in die Vernichtung und sogar Selbstvernichtung getrieben, aber da, wo Franza den Pro-sess ihrer eigenen Vernichtung stellvertretend erlebt, und gleichzeitig bei totaler Identifikation mit den Opfern auch fähig ist ihre Komplizenschaft mitzudenken, erliegt Fanny primär der Kränkung ihrer Eigenliebe, ist aber im Gegensatz zu Franza nicht in der Lage, den Zusammenhang zur universalen Schändung in der Geschichte herzustellen. Und gerade an ihr hat sie wesentlich aktiver teil, als Franza vor und das Ich in Malina nach ihr. Wie die Erzählungen Jugend in einer östereichischen Stadt, wie UnterMördern und Irren, sollte der Einstieg zum Requiem, der bisher immer nur auf sein Ende hin interpretiert worden ist, vielleicht als Bachmanns radikalster Beitrag zur Auseinandersetzung mit der österreichischen NS-Vergangenheit gelesen werden.

Das, was die Vorrede zu Franza leisten soll, konstituiert im Requiem den ersten Satz: «In einer längst vergangenen Zeit, die von unregelmässiger Bedeutung ist, trotz ihres Eingreifens in das Leben aller, die damals gelebt haben, gab es eine Fanny Wischnewski [...] $)^{33}$. Eine solche unmärchenhafte Märchen-Eröffnung klingt nach Programm: geradezu penetrant wird hier gemahnt, dass die Zeit, von der die Rede ist, 
unlautererweise als vergangen abgetan wird, obwohl sie doch in das Leben aller eingegriffen hat. Die Protagonistin Fanny ist doppelt belastet; nicht nur war die Jugendliche deutsche Flakhelferin, ihr Vater war ausserdem ein österreichsicher Faschist, der mit den deutschen Nationalsozialisten paktiert hatte. Im Gegensatz zu Mutter und Tante, die gerne den Selbstmörder zum politischen Helden stilisiert sähen, weiss die Tochter um die Schande des Vaters und beschliesst die für ihre Umwelt glücklicherweise undurchsichtigen politischen Hintergründe auf sich beruhen $\mathrm{zu}$ lassen, indem sie energisch dafür plädiert, einen Schlusstrich unter dieses «unglückselige Geschichtskapitel» zu ziehen. Der Leser ist gewarnt, Fanny gehört von vornherein zu den Strategen des Vergessens.

So steht ihr Treffen mit dem aus der Emigration zurückgekehrten Juden Goldmann, ihr späterer Mann, von Anfang an unter einem ungünstigen Stern. Ihre respektiven Vergangenheiten verbergen sie voreinander, und schauspielern sich durch eine Ehe, die alsbald geschieden wird. Goldmann fährt wieder nach Amerika, dann nach Israel. Als er nach Wien zurückkehrt findet ihn Fanny «sehr verändert». Als er zu reden anfängt: «vermochte (sie) kaum zuzuhören, sie meinte immer, alles müsse sich auf sie beziehen», kommentiert der Erzähler, scheint mir, nicht ohne Vorwurf, «aber nun

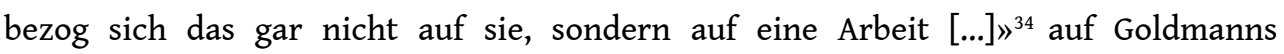
Beschäftigungen mit der Geschichte der Juden. Der Ernst Goldmann, «den Jude sein zu etwas verpflichtet oder getrieben hatte», der dem Eichmann-Prozess beiwohnen wollte, den kennt sie nicht, ja will ihn nicht kennen. Er, Goldmann, holt nach, was er in der direkten Nachkriegszeit versäumt hatte, steigt ein in die Erinnerungsarbeit über eine Vergangenheit, die er vorher ignorieren zu können meinte. Fanny versteht Goldmanns Wandel nicht, erkennt ihn nicht als Aufforderung, ihrer eigenen Verstrickung in der österreichischen NS-Geschichte nachzuforschen. Vielmehr verengt sich ihr Blick hinter private Blessuren, die sie blind machen für die geschichtlichen Zusammenhänge, von der schliesslich auch das Heil ihrer Liebesgeschichte abhängt. Wie Fremde stehen die Österreicherin und der Jude einander gegenüber: sie fühlt sich von ihm ausgeschlossen, er versteht nicht, warum sie nicht versteht. Man möchte in dieser Beschreibung ein Paradigma erkennen, so etwas wie eine möglichst objektive Lagebeschreibung, wie schlecht es um das Verhältnis von Österreichern und Juden im Wien der sechziger Jahre immer noch bestellt ist, eben ein Verhältnis, das selbst im besten Fall keins sein kann, weil jegliche Ver Stehensbereitschaft - auf beiden Seiten - fehlt.

Obwohl sprachlich gesehen das Unbehagen am Kontinuum der NS-Mächtigen mit Marek und Karin im «Fanny-Fragment» für Bachmannsche Verhältnisse geradezu tendenziös veranschaulicht wird, ist die Lesart der Geschichte hier - im Gegensatz zu den zwei anderen Todesartenwerken - nicht linear feministisch ausgerichtet, wonach die Frau per se unschuldiges Opfer, und der Mann per se brutaler Täter ist. Goldmann und Fanny als Mann und Frau sind beide schuldig/unschuldig, und später werden Marek und Karin wiederum als Mann und Frau gleichermassen als ekelerregende Produkte der NS-Barbarei österreichischer und deutscher Provenienz gezeichnet.

17 Auch wenn das Requiem bisher immer nur im Hinblick auf Fannys Vernichtung hin gelesen wurde, scheint es mir wichtig, die Konfigurationen des Anfangs (Fanny vis-à-vis Goldmann) mit zu berücksichtigen, weil sie nicht nur das starre Opfer-Täter Schema durchbrechen, sondern die Geschlechterproblematik differenzieren und damit auch ein anderes Licht auf Bachmanns «Faschismustheorie» werfen. Hier, im Gegensatz zum Franza-Fragment, ist der «öffentliche» Faschismus Ursache für die Entzweiung einer 
privaten Beziehung, und nicht wie bei Franza und Jordan lediglich die Intensivierung eines schon pre-existenten Atavismus zwischen den Geschlechtern.

In «Malina», dem einzig abgeschlossenen Roman, werden die Gewichte wieder in Richtung Eindeutigkeit verlagert. Der männliche Name im Titel (trotz weiblicher Endung) zeichnet verantwortlich für den Kriminalbefund am Ende: «Es war Mord». Geradlinig, wenn auch nicht ohne Ambivalenzen, wird hier - wiederum im Gegensatz zum Requiem - auf extrem verschlüsselte Weise die Geschichte einer Ausmerzung vorgeführt. So liegt der Schwerpunkt hier wieder - nicht wie beim Requiem auf dem NSKontinuum, sondern auf dem Bedeutungsgehalt von Auschwitz. Das ermordete Ich erzählt die Geschichte seiner Ermordung. Selbst ohne Namen, nicht nur, weil es stellvertretend für das Weibliche schlechthin steht, sondern auch weil es zur Namenlosigkeit, zum Nichtleben-können verurteilt ist, weiss es seine mörderischen männlichen Instanzen sehr wohl zu benennen : den Ungarn Ivan, den wir schon in seiner deutschen Fassung als das «Ungeheuer» Hans aus Undine geht kennen, Malina, der bei aller Mehrdeutigkeit seinem Anagramm Animal, einem Raubtier zumal, zu gleichen kommt, und schliesslich den Vater, Hitler und Gott in einem, Urprung und Verkörperung aller männlichen Schreckensherrschaft.

In Malina wird subjektives Erleiden mit «kollektiver Erinnerung» zu einer überzeugenden Synthese miteinander verwoben. Wie schon im Fall Franza verdichtet sich die alptraumartig erlebte Ermordung des Ich in der Metapher des «Friedhofs der ermordeten Töchter» und gipfelt in der Halluzination des Todeslagers. Mit diesem Höhepunkt des Grauens schreibt sich die Ausrottung des weiblichen Ich in die Geschichte des Judeogenozids ein. Wie dies schon andeutungsweise im «Fall Franza» geschah, wird in "Malina», in einem monumentalem Elaborat, die Vernichtung der Frau mit der Vernichtung der Juden metaphorisch zur Deckung gebracht. Nicht Jordan ist der Folterknecht, sondern der Vater:

Die Kammer ist gross und dunkel... Denn es gibt keine Fenster und keine Türen. Mein Vater hat mich eingeschlossen, und ich will ihn fragen, was er vorhat mit mir, aber es fehlt mir der Mut, ihn zu fragen [...] [es gibt] keine öffnungen [...] denn an allen sind schwarze Schläuche angebracht [...] Warum habe ich die Schläuche nicht schon früher bemerkt, denn sie müssen von Anfang an da gewesen sein. Ich war so blind im Halbdunkel und bin die Wände entlanggetappt, um meinen Vater nicht aus den Augen zu verlieren, um die Türen zu finden mit ihm, aber nun finde ich ihn und sage: Die Tür, zeig' mir die Tür. Mein Vater nimmt ruhig einen ersten Schlauch von der Wand $a b$, ich sehe ein rundes Loch durch das es hereinbläst... mein Vater geht weiter, nimmt einen Schlauch nach dem anderen ab, und eh ich schreien kann, atme ich schon das Gas ein, immer mehr Gas. ich bin in der Gaskammer, das ist sie, die grösste Gaskammer der Welt. Man wehrt sich nicht im Gas. Mein Vater ist verschwunden, er hat gewusst, wo die Türe ist und hat sie mir nicht gezeigt, und während ich sterbe stirbt mein Wunsch, ihn noch einmal zu sehen und ihm das Eine zu sagen. Mein Vater, sage ich ihm, der nicht mehr da ist, ich hätte Dich nicht verraten, ich hätte es niemand gesagt $[. ..] »^{35}$.

Die symbolischen Prämissen sind die gleichen wie im Fall Franza: das, was sich hier emphatisch als die "grösste Gaskammer der Welt» präsentiert, ist - wie schon erwähnt - der Raum der Geschichte, der Raum unserer Geschichte. In diesen Raum hat der «Vater», Derivat Hitlers, die «Tochter» eingeschlossen. Er kennt die Tür, weiss einen Fluchtweg, die Tochter aber, lässt er im Gas verenden. Sie hätte ihm gerne noch «das Eine» gesagt, heisst, das Eine gefragt, und versichert ihm, dass sie ihn nicht verraten hätte. 
21 Mir scheint, dass wir es hier mit einem Gleichnis zu tun haben, das nach bildlichen Äquivalenten sucht, um das Trauma der zweiten Generation zu fassen, das sich am deutlichsten im zerstörten Verhältnis der Tätergeneration und ihrer Nachkommenschaft niederschlägt. Die Täter selber verweigern sich der Verantwortung, sie steigen aus der Geschichte aus, fliehen in die Verdrängung, in das Vergessen. Und weil sie sich ihrer Verantwortung verweigern, müssen ihre Nachkommen die Last der Erbschaft auf sich nehmen, eine Erbschaft die sie überwältigt, so wie das Gas die NS-Opfer überwältigt hat ${ }^{36}$. Das «Eine» wollte die Tochter dem Vater abverlangen, das Geständnis seiner geschichtlichen Schuld. Aber er verschliesst sich dem Dialog, setzt stattdessen die Tochter «dem Gas» aus, während er schon die Flucht ergriffen hat, obwohl die schon fast Gestorbene dem schon Geflohenen verspricht, dass sie ihn nicht verraten hätte. Die jahrhundertealte Unterdrückung der Frau durch das Patriarchat, die ja den ganzen Todesartenzyklus bestimmt, wird hier mit einer ganz präzisen zeitgeschichtlichen Aussage aufgeladen: der Gaskammertraum des Ich liest sich nicht zuletzt wie ein «Vaterbuch $»^{37}$. Es ist u.a. auch ein literarisches Modell für die verspätete Auseinandersetzung, bzw. für die gewalttätige Ablehnung einer solchen Auseinandersetzung, zwischen den Generationen.

Bachmanns Auschwitz, ihr Kompendium von Todesarten, wie sie das Projekt nannte, ist - um mit Adorno zu sprechen - das Bewusstsein von ihren Nöten. Ihr Zyklus inszeniert den Krieg gegen das Vergessen. Literatur wird hier nicht nur zu Gedächtnis, sie wird auch zu einer Instanz, die Gedächtnis weckt. Die Wand Auschwitz steht nicht nur zwischen der Österreicherin Fanny und dem Juden Goldmann, sie steht nicht nur zwischen dem Spezialisten für Spätschäden Jordan und dem imaginären Häftling Franza, sie steht auch zwischen den Vätern und ihren Töchtern. Und während die Väter und die Ehemänner, die Brüder und die Geliebten sie noch nutzbar zu machen wissen für sich, schlagen sich die Frauen und die Töchter den Kopf wund an ihr, verschwinden sie in diesen todbringenden Riss der Geschichte, - einziger Ausweg aus der Ausweglosigkeit Auschwitz.

\section{NOTES}

1. - Zitiert wird aus der Werkausgabe, Ingeborg Bachmann Werke, Hg. von Christine Koschel, Inge von Weidenbaum, Clemens Münster (WA Bd. 1-4) Bd. 3, S. 433.

2. - Ebd. S. 403.

3. - $\mathrm{Zu}$ diesem Thema vgl. Irene Heidelberger-Leonard: Ingeborg Bachmann und Jean Améry: Zur Differenz zwischen der Ästhetisierung des Leidens und der Authentizität traumatischer Erfahrung. In: Andrea Stoll (Hg.): Malina, Frankfurt : Suhrkamp 1992, S. 288-300.

4. - Vgl. Hans Höller: «Die Polen-Interviews» Ingeborg Bachmanns und ihre Stellung im Gesamtwerk. In: Österreichisch-Polnische Literarische Nachbarschaft - Uam Poznan 1979, S. 127-135.

5. - In: Ingeborg Bachmann: Wir müssen wahre Sätze finden. Gespräche und Interviews. München: Piper 1982, S. 111.

6. - Ebd. S. 137. 
7. - Ebd. S. 131.

8. - Ebd. S. 142.

9. - WA, Bd. 3, S. 406.

10. - Ebd. S. 341.

11. - Ebd. S. 342.

12. - Ebd.

13. - S. Anm. 3.

14. - WA, Bd. 3, S. 372.

15. - Ebd. S. 379.

16. - Ebd. S. 383.

17. - Ebd. S. 401.

18. - Ebd. S. 404.

19. - Ebd. S. 405.

20. - Ebd. S. 404.

21. - Ebd. S. 407.

22. - Ebd. S. 413.

23. - Ebd. S. 455.

24. - Ebd. S. 456.

25. - WA, Bd. 3, S. 412.

26. - Ebd. S. 407.

27. - Für die Interpretation der Gaskammerträume im Fall Franza und in Malina verdanke ich wertwolle Denkanstösse Holger Gehle, dem ich an dieser Stelle für anregende Auseinandersetzungen danken möchte.

28. - WA, Bd. 3, S. 407.

29. - Ebd. S. 457.

30. - Ebd.

31. - Ebd. S. 458.

32. - Auch wenn die Produktionsgeschichte des Requiem für Fanny Goldmann noch nicht mit Sicherheit rekonstruiert werden kann, sollte man den Fragmentcharakter nicht zum Vorwand nehmen, sich der Kritik, die sich hinter der Fanny/Goldmann Konstellation verbirgt, zu stellen.

33. - WA, Bd. 3, S. 485.

34. - Ebd. S. 493.

35. - Ebd. S. 176.

36. - Diese Analogie scheint mir nicht ganz unproblematisch, weil sie leicht für eine unfreiwillige - Verharmlosung der tatsächlichen Gastod-Opfer gehalten werden kann.

37. - So gelesen bietet sich Malina auch als ein Buch an über «unbewältigte Vergangenheiten» von einer männlichen Täter generation (Vater), die ihr Geschichtserbe verleugnet und ihrer (weiblichen) Nachkommenschaft (Tochter), die an ihrer ererbten, wenn auch nicht selbst verursachten, Schuld zugrunde geht. vgl. dazu Marianne Ufer: Sprachzerstörung und Sprachverstörung. Versuche zur Uberwindung durch Autorinnen der achtziger Jahre. In: Annali della Facolta di Lettere e Filosofia, 1989-1991, S. 197-228. 


\section{RÉSUMÉS}

Im Vordergrund steht die Frage nach dem geschichtlichen Gehalt der Roman-Trilogie, die - trotz ihrer Unabgeschlossenheit - Bachmanns kulturgeschichtliche Entwicklung vorführt, den die Autorin mit der Formulierung vom «Ich in der Geschichte» zur «Geschichte im Ich» fasst. Der Aufsatz prüft die Metaphorik, mit der Bachmann ihr eigenes Geschichtstrauma zu vermitteln sucht. Wie sich in jedem der drei Teile: «Der Fall Franza», «Requiem für Fanny Goldmann» und dem einzig beendeten Roman «Malina» der Rassendiskurs der Nationalsozialisten mit dem heutigen Geschlechterdiskurs überschneidet, wie die Ausmerzung der Juden für Bachmann streckenweise zu einem problematischen Synonym wird für die Ausmerzung der Frau, ist Thema dieser Ausführungen.

Cet article traite principalement le problème du contenu historique de la trilogie romanesque, celle-ci, bien qu'inachevée, présentant l'évolution des attitudes d'Ingeborg Bachmann face à l'histoire, évolution que l'auteur formule en disant qu'elle est passée du « moi dans l'Histoire » à «l'Histoire dans le moi ». Cette contribution étudie le métaphorisme par lequel I. Bachmann essaie de communiquer au lecteur le traumatisme que lui a infligé à elle-même la réalité historique. Il s'agit de montrer comment, dans chacune des trois parties : "Le cas Franza ", « Requiem pour Fanny Goldmann » et le roman «Malina ( (le seul à être achevé), le discours raciste des nazis se recoupe avec le discours sexiste d'aujourd'hui : pour I. Bachmann, l'élimination des Juifs est par certains aspects assimilable du fait de sa problématique à la destruction de la femme.

\section{AUTEUR}

\section{IRENE HEIDELBERGER-LEONARD}

Université Libre de Bruxelles 